\title{
Evaluación Inclusiva e Intercultural: Una mirada a la educación en el pueblo Waorani
}

\section{Inclusive and Intercultural Evaluation: A look at education in the Waorani people}

Yépez Rosero Alexandra

Hidalgo Mantilla Geomar Dinora

Procel Guerra Betty Shadira

Pontificia Universidad Católica del Ecuador, Ecuador

Autor para correspondencia: syepez838@puce.edu.ec

Fecha de recepción: 23 de enero 2018 - Fecha de aceptación: 26 de febrero de 2018

Resumen: Esta investigación hace un recorrido del anclaje de la inclusión y la interculturalidad en el ámbito de la educación general básica en el Ecuador, revisando los principales documentos que sustentan las políticas y las directrices y a partir de esta revisión se llega a la reflexión de la realidad observada en el Centro Comunitario de Educación Intercultural Bilingüe, ubicado en el Parque Nacional Yasuní, que da atención a escolares de la nacionalidad waorani, con el propósito de revelar el conocimiento fragmentado que se mantiene sobre las temáticas en cuestión. El artículo pretende dilucidar si la aplicación de instrumentos formulados para medir destrezas, habilidades, competencias necesarias en el desempeño escolar, requieren ser contextualizados, tomando en cuenta la diversidad sociocultural y el principio de interculturalidad planteado en la Constitución del 2008. Esta reflexión convoca a observar los resultados que se obtienen de la aplicación de las pruebas CUMANIN y AE-P, baterías estandarizadas y con alta confiabilidad, construidas desde contextos que no toman en cuenta las realidades socioculturales. Los resultados de las pruebas aplicadas no reflejan las habilidades o destrezas manifestadas por los niños Waorani. De allí la convocatoria a que cualquier tipo de evaluación deberá ser previamente adaptada considerando los contextos socioculturales, para hablar de inclusión y respeto a la diversidad.

Palabras claves: evaluación; inclusión; interculturalidad; educación

\begin{abstract}
The following investigation gives a bird's eye view of social Inclusion and Interculturality in the area of General Basic Education in Ecuador. This article reflects upon the reality that the Community Center for Intercultural and Bilingual Education, located in the Yasuní National Park, undergoes by reviewing important documents that contain policies and regulations which reveal the fragmented know- how about social inclusion and interculturality. The center attends to Waorani schoolchildren. What intends to be clarified is if the aplication of instruments intended to measure knowledge, skills and competences, necessary for better school performance, need to be contextualized and take into consideration sociocultural diversity and the principle of interculturality proposed in the Ecuadorian Constitution of 2008. The results of the application of a battery of standarized CUMANIN y AE-P tests were observed, which although are highly reliable, do not take into consideration social cultural contexts. Such results do not convey the
\end{abstract}


skills and competencies that Waorani children have thus, the need for tests to be previously adapted to social cultural contexts in order to abide by the principles of social inclusion, interculturality and diversity.

Key words: testing; social inclusion; interculturality; education

\section{Introducción}

La investigación que condujo a la publicación del presente artículo se realizó durante un año y medio a partir de octubre del año 2016 en la escuela IKA ubicada en la parroquia Alejandro Labaka de la provincia de Orellana en la Amazonía ecuatoriana, el trabajo de campo se ha enfocado en los niños de cuatro comunidades que pertenecen a la nacionalidad waorani: Guiyero, Ganketapare, Peneno, Timpoka.

El contacto que los investigadores han realizado con estas comunidades se da gracias al impulso que la Pontificia Universidad Católica del Ecuador PUCE proporciona para investigaciones en el área social de esta zona donde se encuentra la Estación Científica Yasuní de esta universidad.

El haber ingresado a observar una temática como la inclusión en el ámbito educativo responde a la necesidad de mirar a la diversidad en distintos aspectos y más aún a la problemática que está surgiendo frente a esa diversidad que muchas veces recae en la necesidad de incluir y tantas veces más en una realidad de exclusión, como menciona Manzano (2001) en Echeita (2014)"luchar contra todas las formas de exclusión y discriminación para promover la integración y participación de los grupos más vulnerables en la vida económica y social” pag.78

De la misma manera una problemática contemporánea que se llega en unas ocasiones a evidenciar y en otras, pasa desapercibida, según Echeita (2014) la evaluación y selección de los estudiantes se convierten en parámetros de exclusión en los sistemas educativos; desencadenando en riesgo de llegar al fracaso escolar y con ello a la exclusión social. pag.81

En el Ecuador, el Ministerio de Educación oficializó a partir del 2008, la implementación de las pruebas SER ECUADOR, para la evaluación del desempeño de los estudiantes, (Ministerio de Educación, 2008), pruebas que fueron aplicadas a todos los alumnos del país sin considerar sus particularidades. Así, en la investigación se utilizan pruebas estandarizadas en los niños waorani de la escuela IKA a partir de las cuales se identifican las dificultades a la hora de aplicar y obtener resultados.

El trabajo deja abierta la posibilidad de adaptar los instrumentos de medición con los parámetros identificados en el marco de la inclusión educativa, de acuerdo con lo que plantea Arnaiz (2003) citado en Rosano (2007).

Para abordar esta necesidad de evaluar tomando en cuenta los contextos socioculturales, es pertinente mencionar los principios de estas variables manejadas en la investigación:

\section{Evaluación}


La evaluación, inclusión e interculturalidad son temas que en la actualidad en el Ecuador cobran importancia y se conjugan con el tema de calidad educativa; de allí la aplicación de una serie de instrumentos para evaluar esta pretendida calidad.

La evaluación al desempeño del estudiante, planteada en Ecuador pretende, a través de la aplicación de varios instrumentos de evaluación, medir los conocimientos, habilidades, destrezas y valores desarrollados, como resultado del proceso educativo y su aplicación en la vida cotidiana.

Con las pruebas se aplican cuestionarios de contexto para evaluar los factores intraescolares y extraescolares que inciden en el aprendizaje de los estudiantes. Los resultados permitirán hacer seguimiento a la calidad de la educación y con ello proponer estrategias de mejoramiento de la enseñanza y el aprendizaje que brinda el sistema educativo nacional. (Ministerio de Educación 2008)

La evaluación, inclusión e interculturalidad son temas que en la actualidad en el Ecuador cobran importancia y se conjugan con el tema de calidad educativa; de allí la aplicación de una serie de instrumentos para evaluar esta pretendida calidad.

La inclusión dentro del marco legal constituye un derecho de todas las personas, que implica respetar la diversidad, sin excepciones.

De acuerdo con lo establecido por Ainscow y Booth, socializado por la UNESCO (2005):

[...] La educación inclusiva, más que un tema marginal que trata sobre cómo integrar a ciertos estudiantes a la enseñanza convencional, representa una perspectiva que debe servir para analizar cómo transformar los sistemas educativos y otros entornos de aprendizaje, con el fin de responder a la diversidad de los estudiantes. El propósito de la educación inclusiva es permitir que el profesorado y estudiantes se sientan cómodos ante la diversidad y la perciban no como un problema, sino como un desafí y una oportunidad para enriquecer el entorno de aprendizaje. (UNESCO, 2005, p. 15., los énfasis son nuestros)

Uno de los principios de la inclusión habla de la igualdad de derechos, este se refiere a que todos deben tener las mismas oportunidades de educación de calidad, entendida la calidad de la enseñanza como planificar, proporcionar y evaluar el currículo óptimo para cada alumno, en el contexto de una diversidad de individuos que aprenden.(Wilson, J.D.,1992:34)que implique el respeto a las diferencias individuales, (contexto social, etnia, etc), como fundamento de la equidad que confluye en el goce de oportunidades.

Hablar de interculturalidad en educación es pensar en los principios que lo fundamentan, parafraseando a la UNESCO, la educación intercultural respeta y enseña, conocimientos, actitudes y las competencias culturales para contribuir al entendimiento y solidaridad entre individuos. De allí que hablar de interculturalidad en educación media es pensar en estos principios: tomado de Directrices de la UNESCO sobre la educación intercultural (http://unesdoc.unesco.org/images/0014/001478/147878s.pdf). 
$\checkmark$ Principio I: La educación intercultural respeta la identidad cultural del educando impartiendo a todos, una educación de calidad que se adecúe y adapte a su cultura.

$\checkmark$ Principio II: La educación intercultural enseña a cada educando los conocimientos, actitudes y las competencias culturales necesarias para que pueda participar plena y activamente en la sociedad.

$\checkmark$ Principio III: La educación intercultural enseña a todos los educandos los conocimientos, actitudes y las competencias culturales que les permiten contribuir al respeto, el entendimiento y la solidaridad entre individuos, entre grupos étnicos, sociales, culturales y religiosos y entre naciones. (pág. 35)

En la constitución del 2008 de la República se reconoce al Ecuador como un país [...] intercultural y plurinacional. Esta declaratoria conlleva a la promulgación de la Ley Orgánica de Educación Intercultural, direccionada para el funcionamiento del Sistema de Educación General Básica y Bachillerato; en esta Ley, en el Título I, art. 1.- Ámbito. - La presente Ley garantiza el derecho a la educación, determina los principios y fines generales que orientan la educación ecuatoriana en el marco del Buen Vivir, la interculturalidad y la plurinacionalidad; así como las relaciones entre sus actores.

En este contexto donde se quiere erradicar la discriminación se implementan evaluaciones estandarizadas, es cuando surge la pregunta ¿se puede evaluar de la misma manera y con los mismos instrumentos a aquellos educandos que en el sistema mismo han sido diferenciados por su riqueza cultural? ¿Acaso los instrumentos de evaluación manejan criterios en los que se cumplen los principios de la inclusión y la interculturalidad? ¿O, por el contrario, estamos discriminando a los niños y niñas indígenas, quienes no superan las pruebas debido a que por su contexto socio cultural poseen otro tipo de habilidades que las que pretendemos medir?

\section{Metodología}

\section{Diseño de la investigación}

La investigación realizada durante 15 meses en los años 2016 y 2017, aprobada el 11 de octubre del 2016 por el Comité de Ética de la Investigación en Seres Humanos - PUCE.

La investigación es de tipo exploratoria, estudio de caso, con un enfoque mixto, cuyo objetivo fue determinar la dificultad para aplicar pruebas estandarizadas en un contexto socio cultural particular y evaluar con ellas destrezas, habilidades y valores desarrollados en los niños de educación general básica del Centro Comunitario Intercultural Bilingüe IKA, ubicado en la zona del Parque Nacional Yasuní.

La primera fase de la investigación, para la recolección de datos, recurrió a la etnografía, se realizó entrevistas a los padres o representantes de los niños waoriani de la comunidad Ika. Para recabar datos de importancia sobre hitos evolutivos, formas de crianza, datos escolares y 
sociales. En una segunda fase se aplicó el Test CUMANIN y el AE-P, La tercera fase consistió en la tabulación e interpretación de los datos obtenidos en las pruebas.

\section{Participantes}

Se trabajó con estudiantes matriculados en la Escuela IKA, durante el año lectivo 20162017; cuyos padres firmaron el consentimiento informado aceptando la investigación.

El CUMANIN se aplicó a 12 niños de primero y segundo año de educación general básica en un rango de edad de 5 a 6 años 11 meses de los cuales 5 son mujeres y 7 hombres. El AE-P se aplicó a catorce niños de tercero cuarto, quinto y sex to año de educación general básica en un rango de edad de nueve años a 12 años 6 meses de los cuales 6 son hombres y 8 mujeres.

No se tuvo criterios de exclusión por cuanto el universo está conformado por todos los niños matriculados en los respectivos grados.

\section{Instrumentos y técnicas utilizadas para recolección de datos}

Se utilizaron dos instrumentos: el primero fue la prueba: CUMANIN que es un cuestionario que valora la madurez neuropsicológica infantil, está constituido por cinco pruebas (psicomotricidad, lenguaje articulatorio, lenguaje expresivo, lenguaje comprensivo, estructuración espacial, visopercepción, memoria icónica, ritmo) y por cuatros subpruebas (Fluidez verbal, atención, lectura, escritura con puntuación de un punto por cada ítem. (ver tabla 1) y A-EP (evaluación de la autoestima en la educación primaria autoconcepto, (ver tabla No 2)

Para ingresar a las comunidades y trabajar en la escuela fue necesario el apoyo de cinco jóvenes waorani bachilleres que se ofrecieron voluntariamente a acompañar y colaborar con la traducción del waotededo a español o viceversa. Los investigadores también contaron con la ayuda de Juan Carlos Armijos, profesor de la PUCE, quien, por tener acceso desde hace veinte años, hablar el waotededo y haber sido aceptado por estas comunidades facilita el contacto con los miembros de estas poblaciones.

Para la aplicación de estas pruebas se recurrió a los intérpretes waorani, que facilitaron la comunicación y comprensión de las instrucciones. (Cuando los niños así lo solicitaban)

El análisis de la información, tomó como referencia baremos establecidos para cada prueba, se recurrió a la estadística descriptiva, para identificar medidas de tendencia central.

Para el análisis de las dificultades encontradas tanto en la aplicación como en la evaluación se recurrió al registro de la observación.

\section{Tabla 1: Ficha técnica CUMANIN:}

\begin{tabular}{ll}
\hline Datos & Descripción \\
\hline Nombre & \\
Original: & Cuestionario de Madurez Neuropsicológica Infantil (CUMANIN) \\
Autores: & José Antonio Portellano Pérez, Rocío Mateos Mateos y Rosario Martínez Arias. \\
\hline
\end{tabular}




\begin{abstract}
Procedencia: $\quad$ Madrid, TEA Ediciones, 2009
Aplicación: Individual

Ámbito de

Aplicación: $\quad$ Sujetos con edades comprendidas entre 36 meses y 78 meses.

Duración: $\quad$ Entre 30 y 50 minutos.

Evaluar el grado de madurez neuropsicológica alcanzada en relación con su edad cronológica.

Constatar las consecuencias que tiene el funcionamiento alterado del sistema nervioso sobre la conducta y las funciones cognitivas. Útil para evaluar Psicomotricidad, Lenguaje articulatorio,

Finalidad: $\quad$ Lenguaje comprensivo, Lenguaje expresivo, Estructuración Espacial, Visopercepción, Memoria Icónica y Ritmo; además se puede evaluar, atención, fluidez verbal, lectura, escritura y lateralidad, con la finalidad de conocer los mecanismos neuropsicológicos que pueden estar alterados en niños de edad preescolar, los cuales pudieran traducirse en futuros casos de dificultad de aprendizaje.

Tipificación Puntuaciones centiles en las escalas y sus agrupaciones y conversión de la puntuación total en un índice de desarrollo (CD).

Descripción El test está conformado por ocho escalas principales y cinco adicionales que permiten la del test: $\quad$ exploración neuropsicológica y la obtención de un cociente del desarrollo.

Se recomienda utilizar un espacio amplio, con adecuadas condiciones de iluminación y con el menor número de obstáculos posible; se necesita también una mesa y silla. Se seguirá siempre el Forma de orden indicado, iniciando el examen por la escala de psicomotricidad y finalizando por la de Aplicación: ritmo. Se aplicarán todas las escalas a todos los niños, con excepción de las de lectura y escritura que se aplicarán a niños mayores de 5 años si ya poseen estas habilidades. Se debe hacer ver la prueba como un juego divertido. Se recomienda la utilización de reforzadores positivos como caramelos, dibujos, cromos; además de refuerzos verbales que elogien sus resultados.
\end{abstract}

Fuente: Portellano, J., Mateos, R., Martínez, R. Tapia, A. Granados, Ma. (2009)

Tabla 2: Ficha técnica A-EP:

\begin{tabular}{|c|c|}
\hline Datos & Descripción \\
\hline Nombre & \\
\hline Original: & Cuestionario para la evaluación de la autoestima en Educación Primaria A-EP \\
\hline Autores: & Ramos, Giménez, Muñoz-Adell, Lapaz \\
\hline Procedencia: & TEA Edic., 2006, Madrid \\
\hline Aplicación: & Individual o colectiva de acuerdo con la edad. \\
\hline Ámbito de & \\
\hline Aplicación: & Sujetos con edades comprendidas entre los 9 y los 12 años \\
\hline Finalidad: & $\begin{array}{l}\text { Este cuestionario tiene como finalidad la medición global de la autoestima, entendiéndose esta } \\
\text { como la percepción que cada individuo tiene de sí mismo y que se forma a partir de las } \\
\text { experiencias y relaciones con el entorno. }\end{array}$ \\
\hline Baremación: & $\begin{array}{l}\text { La baremación que se maneja es la escala percentil (PC) y de puntuaciones típicas derivadas } \\
\text { (PTD), con una media de } 50 \text { y una desviación típica de } 10 .\end{array}$ \\
\hline $\begin{array}{l}\text { Descrpición } \\
\text { del test: }\end{array}$ & $\begin{array}{l}\text { Las preguntas son breves, directas, con un vocabulario comprensible adaptado a la edad y están } \\
\text { acompañadas de ilustraciones. La muestra de tipificación para la construcción del test incluyó } \\
\text { niños de nivel social y cultural variado, muchos de ellos de origen marroquí o proveniente de otros } \\
\text { países del norte de África que viven en España. }\end{array}$ \\
\hline
\end{tabular}

Fuente: Ramos, R. Gimenez, A. Muñoz, A. La paz, Ma. (2006). 


\section{Resultados}

Se muestra la tabla 3 con los resultados del test CUMANIN de los que se desprende que las sub-pruebas con menor puntuación en el promedio de los percentiles son: Atención con 5.7 y Lenguaje Comprensivo con 5.5 y la sub-prueba con mayor puntuación es memoria icónica con un promedio de 61.7.

Respecto a la autoestima, se aplicó el cuestionario A-EP, de forma individual, a quince niños, cuyas edades se hallan comprendidas entre los nueve y doce años. El promedio grupal se ubica en el rango de Autoestima dentro de la media. Ver tabla 4 
Tabla 3: Resultados obtenidos del Cuestionario CUMANIN de las 7 escalas principales y 2 complementarias de acuerdo con Centiles. Aplicado a 12 niños y niñas de 5 a 6 años 11 meses, de la escuela IKA.

\begin{tabular}{|c|c|c|c|c|c|c|c|c|c|c|c|c|c|}
\hline $\begin{array}{l}\text { No } \\
\text {. }\end{array}$ & $\begin{array}{l}\text { Códig } \\
\text { o }\end{array}$ & $\begin{array}{l}\text { Sex } \\
0\end{array}$ & Edad & $\begin{array}{l}\text { Psicomotrocida } \\
\text { d }\end{array}$ & $\begin{array}{l}\text { Lenguaje } \\
\text { Articulatori } \\
\text { o }\end{array}$ & $\begin{array}{l}\text { Lenguaje } \\
\text { Expresiv } \\
\text { o }\end{array}$ & $\begin{array}{l}\text { Lenguaje } \\
\text { Comprensiv } \\
\text { o }\end{array}$ & $\begin{array}{l}\text { Estructuració } \\
\text { n Espacial }\end{array}$ & $\begin{array}{l}\text { Viso } \\
\text { percepció } \\
\mathrm{n}\end{array}$ & $\begin{array}{l}\text { Memori } \\
\text { a } \\
\text { Icónica }\end{array}$ & $\begin{array}{l}\text { Ritm } \\
\text { o }\end{array}$ & $\begin{array}{l}\text { Fluide } \\
\text { Z } \\
\text { Verbal }\end{array}$ & $\begin{array}{l}\text { Atenció } \\
\mathrm{n}\end{array}$ \\
\hline & $1-* \mathrm{D}-$ & & & & & & & & & & & & \\
\hline 1 & 2 & M & 5 años 2 meses & 5 & 20 & 60 & 2 & 99 & 20 & 90 & 4 & 85 & 3 \\
\hline 2 & $1-7-2$ & M & 5 años 2 meses & 5 & 30 & 25 & 2 & 20 & 50 & 90 & 15 & 25 & 5 \\
\hline 3 & $1-*_{-}$ & $\mathrm{V}$ & 5 años 9 meses & 4 & 60 & 20 & 1 & 20 & 15 & 80 & 2 & 15 & 2 \\
\hline 4 & $1-8-3$ & $\mathrm{~V}$ & 5 años 2 meses & 5 & 35 & 4 & 2 & 15 & 20 & 15 & 15 & 25 & 3 \\
\hline 5 & $1-28-1$ & $\mathrm{~V}$ & $\begin{array}{l}5 \text { años } 0 \text { meses } \\
5 \text { años } 10\end{array}$ & 10 & 45 & 5 & 10 & 3 & 55 & 90 & 25 & 65 & 4 \\
\hline 6 & $1-33-3$ & M & meses & 45 & 60 & 20 & 40 & 10 & 10 & 20 & 35 & 97 & 20 \\
\hline 7 & $2-3-2$ & $\mathrm{~V}$ & 6 años 4 meses & 10 & 15 & 20 & 1 & 30 & 40 & 40 & 2 & 25 & 5 \\
\hline 8 & $2-5-2$ & $\mathrm{~V}$ & $\begin{array}{l}6 \text { años } 9 \text { meses } \\
6 \text { años } 11\end{array}$ & 4 & 25 & 20 & 1 & 15 & 65 & 80 & 20 & 10 & 4 \\
\hline 9 & $2-6-1$ & M & meses & 10 & 15 & 3 & 1 & 10 & 50 & 40 & 2 & 20 & 5 \\
\hline 10 & $2-24-4$ & $\mathrm{~V}$ & 5 años 8 meses & 10 & 40 & 20 & 4 & 20 & 40 & 95 & 20 & 35 & 5 \\
\hline 11 & $2-26-1$ & M & $\begin{array}{l}6 \text { años } 5 \text { meses } \\
5 \text { años } 11\end{array}$ & 10 & 10 & 3 & 1 & 20 & 10 & 60 & 2 & 5 & 2 \\
\hline 12 & $2-15-1$ & $\mathrm{~V}$ & meses & 10 & 20 & 20 & 1 & 25 & 40 & 40 & 35 & 40 & 10 \\
\hline \multicolumn{4}{|c|}{ Media del Grupo } & 10,7 & 32,5 & 18,3 & 5,5 & 23,9 & 34,6 & 61,7 & 14,8 & 37,3 & 5,7 \\
\hline
\end{tabular}

Datos recabados por los autores, noviembre 2016 
Tabla 4: Resultados obtenidos en el Cuestionario A-EP en puntuaciones típicas derivadas y percentiles de catorce niños de nueve a doce años, de la escuela IKA.

\begin{tabular}{|c|c|c|c|c|c|c|c|}
\hline $\mathbf{N}^{\circ}$ & Código: & 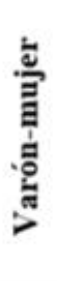 & 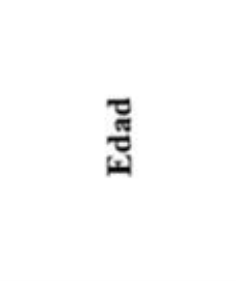 & 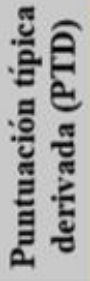 & 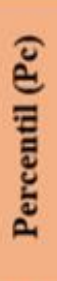 & 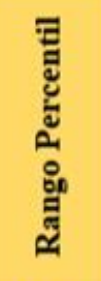 & Interpretación \\
\hline 1 & $3-14-4$ & V & 9 años 2 meses & 52 & 64 & $41-65$ & Autoestima dentro de la media \\
\hline 2 & $3-31-3$ & M & 11 años 2 meses & 52 & 64 & $41-65$ & Autoestima dentro de la media \\
\hline 3 & $4-33-2$ & M & 9 años & 47 & 45 & $41-65$ & Autoestima dentro de la media \\
\hline 4 & $4-21-3$ & M & 9 años 2 meses & 54 & 74 & $66-84$ & Alta autoestima \\
\hline 5 & $4-5-1$ & M & 9 años 7 meses & 52 & 64 & $41-65$ & Autoestima dentro de la media \\
\hline 6 & $4-1-1$ & M & 11 años 1 mes & 45 & 37 & $15-40$ & Autoestima por debajo de la media \\
\hline 7 & $4-10-2$ & M & 12 años 2 meses & 41 & 25 & $15-40$ & Autoestima por debajo de la media \\
\hline 8 & $5-3-1$ & M & 10 años & 47 & 45 & $41-65$ & Autoestima dentro de la media \\
\hline 9 & $5-24-2$ & V & 11 años 7 meses & 43 & 32 & $15-40$ & Autoestima por debajo de la media \\
\hline 10 & $5-14-3$ & V & 12 años 4 meses & 52 & 64 & $41-65$ & Autoestima dentro de la media \\
\hline 11 & $5-11-1$ & M & 12 años 11 meses & 54 & 74 & $66-84$ & Alta autoestima \\
\hline 12 & $6-33-1$ & V & 11 años 11 meses & 34 & 11 & $1-14$ & Muy baja autoestima \\
\hline 13 & $6-21-2$ & V & 11 años 2 meses & 54 & 74 & $66-84$ & Alta autoestima \\
\hline 14 & Sin código & V & 10 años 4 meses & 41 & 25 & $15-40$ & Autoestima por debajo de la media \\
\hline \multicolumn{4}{|c|}{ Media del grupo } & 48 & 45 & $41-65$ & Autoestima dentro de la media \\
\hline
\end{tabular}

Datos recabados por los autores, febrero y marzo de 2017.

\section{Discusión}

La educación en el Ecuador pretende ser inclusiva y estar bajo los principios de la interculturalidad, aspectos que son amparados por la Constitución del 2008 y la inclusión dentro del marco legal constituye un derecho de todas las personas, que implica respetar la diversidad, sin excepciones, propuesto por la UNESCO.

Estos pretendidos anhelos pierden peso al momento de aplicar herramientas estandarizadas de evaluación en contextos diversos y diferentes como se evidencia en los resultados de este trabajo:

En este contexto donde se quiere erradicar la discriminación se implementan evaluaciones estandarizadas, es cuando surge la necesidad de responder algunas preguntas: ¿se puede evaluar de la misma manera y con los mismos instrumentos a aquellos educandos que en el sistema mismo han sido diferenciados por su riqueza cultural? Contestando a la pregunta se afirma que es imposible evaluar con instrumentos estandarizados, así un ejemplo claro es el observado en la prueba AE-P que pretende medir la autoestima, como categoría occidental, en los waorani no se manifiesta de la misma manera, pero sí se puede encontrar parámetros que podrían estar dentro de esta categoría. 
¿Acaso los instrumentos de evaluación manejan criterios en los que se cumplen los principios de la inclusión y la interculturalidad? ¿O, por el contrario, estamos discriminando a los niños y niñas indígenas, quienes no superan las pruebas debido a que por su contexto socio cultural poseen otro tipo de habilidades que las que pretendemos medir? Los instrumentos aplicados para evaluar no manejan estos principios, son descontextualizados ya que la oralidad en los waorani no se toma en cuenta para ningún tipo de evaluación por cuanto el evaluador maneja códigos escritos, mientras que el waorani tiene códigos orales con significados que en muchas ocasiones son distintos.

La dificultad al aplicar estas pruebas fue principalmente el lenguaje utilizado por el examinador, que limita la comprensión de los examinados debido a que su lengua materna es el waotededo.

Otro aspecto que no se enmarca en la inclusión y la interculturalidad a la hora de evaluar es el tiempo y el ritmo de actividades, puesto que en los waorani el reloj no es el instrumento que determina el tiempo; son otros parámetros de medición como es el caso de cazar, pescar, ir a la chacra, etc. Actividades que satisfacen necesidades y que no están sujetas a horarios ni calendarios, sino que se basan en el conocimiento práctico que puede ser considerado como saberes. bajos.

Como se evidencia en el resultado de la sub-prueba de ritmo, los niños obtienen puntajes

\section{Conclusiones}

Los resultados en las pruebas no tienen un fin diagnóstico ni reflejan la realidad debido a que no se encuentran adaptadas al contexto socio-cultural ni lingüístico de los waorani. Sin embargo, permiten analizar la importancia de tomar en cuenta los principios de la inclusión y la interculturalidad al aplicar cualquier tipo de baterías que pretendan medir habilidades, destrezas y valores desarrollados en los niños.

De esta forma los datos obtenidos en cada una de las escalas del CUMANIN, manifiestan un desempeño global que oscila entre Medio Bajo y Bajo, resultados que no reflejan la realidad observada.

En cuanto al cuestionario AE-P los enunciados planteados a través de adjetivos en algunos ítems no contemplan la inclusión en referencia al respeto a la diversidad y a las características individuales ni se ajustan al reconocimiento de la pluriculturalidad ni consideran los valores construidos por esta cultura.

Los resultados de la investigación conducen a la necesidad de aplicar pruebas contextualizadas con indicadores de medición de acuerdo con las competencias culturales y no una simple adaptación a las necesidades de la cultura desde donde se generan las pruebas. 
Si hablamos de inclusión e interculturalidad debemos partir de las competencias propias de la cultura necesarias para su desarrollo, mas no de aquellas competencias que según la visión occidental colaboran a la educación.

Asimismo, los puntajes bajos obtenidos por los niños indígenas en las pruebas estandarizadas y descontextualizadas podrían fortalecer el etnocentrismo, característico de ideologías que consideran a su grupo cultural superior a otros, desprendiéndose de esta forma de pensar prejuicios discriminatorios.

La evaluación entendida como el proceso para medir "algo" y compararlo con "algo" se lo aplica entre situaciones que son abstractas, que se pronuncian como principios o leyes (inclusión e interculturalidad) con situaciones que son concretas (la práctica cotidiana).

La investigación abre puertas a futuros trabajos en el ámbito educativo específicamente en la evaluación y sus adaptaciones.

\section{Agradecimientos}

El agradecimiento va dirigido a la Pontificia Universidad Católica del Ecuador (PUCE-Q) por el financiamiento del Proyecto de Investigación PUCE 2016: "Escuela Waorani: Entrelazando Educación y Cultura” el mismo que sirvió de base para este artículo.

También a la Estación Científica Yasuní de la (PUCE) por la acogida y la logística brindada al equipo de investigadores.

Al personal de la escuela IKA que abrieron sus puertas para proceder con la investigación.

A las comunidades waorani de Timpoka, Peneno, Ganketapare y Guiyero que nos permitieron ingresar a su mundo y aprender de la riqueza cultural.

A Juan Carlos Armijos por su entrega y cariño con los niños waorani de la escuela IKA que nos permitió acceder a las familias de una manera menos invasiva, no como kowori (gente extraña).

A Carol Caldas estudiante de Psicología de la PUCE, por su colaboración desinteresada en la aplicación de las pruebas. A Fernanda Pazmiño y Verónica Zambrano por el trabajo realizado en la aplicación y calificación de las baterías de las pruebas consideradas para este artículo.

La autoría del artículo pertenece a: Mtr. Alexandra Yépez, Mtr. Shadira Procel, Mtr. Dinora Hidalgo. Las autoras están de acuerdo en el orden propuesto para la aparición dentro del artículo.

\section{Bibliografía}


Alonso, M.J \& Araoz, I. (2011) El impacto de la convención internacional sobre los derechos de las personas con discapacidad en la legislación educativa española. Madrid: CERMI/ Ediciones Cinca. Colección ONU, nº 6.

Alto Comisionado de las Naciones Unidas para los Derechos Humanos, 2002, Declaración y Programa de Acción de Durban Conferencia Mundial contra el Racismo, la Discriminación Racial, la Xenofobia y las Formas Conexas de Intolerancia. Panamericana Formas e Impresos S.A. Bogotá, Colombia

Booth, T., Ainscow, M., \& Kingston, D. (2006). Index para la inclusión: desarrollo del juego, el aprendizaje y la participación en educación infantil (Trad. y adaptación al castellano F.González-Gil, M. Gómez-Vela, C. Jenaro). Bristol: CSIE.http://www.eenet.org.uk/resources/docs/Index\%20EY\%20Spanish.pdf

Constitución De La Republica Del Ecuador, 2008. Registro Oficial No 449: 20-oct-2008, Ecuador

Echeita, G.(2014). Educación para la inclusión o educación sin exclusiones. Bogotá: Narcea

Garza Vizcaya, Eduardo L. de la, La evaluación educativa. Revista Mexicana de Investigación Educativa [en línea] 2004, IX (octubre-diciembre) : [Fecha de consulta: 2 de diciembre de 2017] Disponible en:<http://www.redalyc.org/articulo.oa?id=14002302> ISSN 1405-6666

Hurtado, Jacqueline. (2005) Metodología de la investigación. Una comprensión holística, Caracas, ed.

LOEI. Ley Orgánica de Educación Intercultural. Registro Oficial № 417, 30 de marzo de 2011, Quito.

Ministerio de Educación del Ecuador. 2008. Resultados pruebas censales Ser Ecuador 2008. Recuperado https://educacion.gob.ec/wpcontent/uploads/downloads/2012/08/resultadoPruebasWEB.p df

Portellano, J., Mateos, R., Martínez, R. Tapia, A. Granados, Ma. (2009). Cuestionario de Madurez Neuropsicológica Infantil, Madrid, TEA ed

Ramos, R. Gimenez, A. Muñoz, A. Lapaz, Ma. (2006). Cuestionario para la evaluación de la autoestima en educación primaria, Madrid, TEA, ed

Rosano, Santiago (2007) El camino de la inclusión educativa en Punta hacienda.

UNESCO. $\quad$ Directrices la sobre educación intercultural (http://unesdoc.unesco.org/images/0014/001478/147878s.pdf). Sector Educación Francia (recuperado 02 diciembre 2017) 